\title{
The Gastroenterological Society of Australia
}

The annual general meeting and scientific session of the Gastroenterological Society of Australia was held in Sydney on 27-28 May 1968. The President, Dr P. J. Parsons, was in the Chair.

Sir William Morrow and Professor C. C. Booth were elected to Honorary Membership.

The following new members were elected by Council: Dr M. H. Alp, Mr E. V. Barling, Dr B. R. Beveridge, Dr T. D. Bolin, Mr A. M. Clarke, Mr M. C. Douglas, Dr D. C. Glenn, Mr E. Hennessy, Dr D. J. B. St John, Mr D. R. Kemp, Dr G. M. McLeod, Dr J. G. Morris, Mr P. B. Nelson.

The following are abstracts of papers presented at the Scientific Session.

GASTROINTESTINAL HAEMORRHAGE, GASTRIC ULCER, AND ASPIRIN ABUSE

J. M. DUGGAN This study concerns an examination of 1,634 admissions to the Royal Newcastle Hospital from 1954-1964 for acute upper gastrointestinal haemorrhage, with particular reference to age and sex incidence, site of lesion, and the influence of previous aspirin intake on these. The data were extracted onto punch cards and sorted electronically.

Of the 1,081 patients with peptic ulcer, the male-female ratio was $8: 3$ and the duodenal-gastric ulcer ratio was $5: 3$. Compared with other series there is an excess of gastric ulcers in women, in whom they outnumber duodenal ulcers by $3: 2$. The age frequency distribution curve in females with gastric ulcer is unique in being bimodal with peaks at ages 30-49 and 60-69. This confirms the presence of an epidemic of gastric ulcer in younger women in this community, previously noted elsewhere. The peak at age $30-49$ is shown to be associated with a high rate of aspirin intake, an association not seen in other groups in relation to age, sex, and site of lesion.

In those admitted with bleeding and no radiological lesion, the 'acute lesion' group, two-thirds had had recent aspirin, but in only one fifth was this not part of a continuing habit of aspirin taking.

The evidence supports the author's previous hypothesis that the excessive mortality and morbidity from gastric ulcer in younger women in eastern Australia is related to aspirin abuse.

\section{ACID SECRETION BY THE EVERTED RAT STOMACH}

R. BALNAVE AND P. E. BAUME Over the years it has been the aim of many workers involved in studying gastric secretion to find suitable preparations which will allow certain aspects of the gastric secretory process to be studied specifically. In Australia it is difficult to get an adequate supply of toads and for this reason it is difficult to construct systems using single layers of gastric mucosa from these animals. Accordingly, for experiments involving studies in vitro of gastric mucosa it is necessary to seek an alternative system and for this method we have carried out a number of experiments to evaluate the everted rat stomach preparation which was described in 1965 by Dikstein and Sulman from Israel.

The method involves removal of the stomach from a freshly sacrificed rat, eversion of the stomach through a small incision in the fundus, washing of the stomach to remove any food debris, and suspension of the stomach in a suitably gassed medium for a set collection period.

It has been possible to demonstrate that acid secretion occurs from such a preparation. It appears that the nature of the oxygen mixture used is not critical for subsequent secretion but that the nature of the bathing medium is of critical importance. It was found that a molarity of $0.25 \mathrm{M}$ was most suitable, that a buffered medium was more suitable than an unbuffered one, and that the preparation was exquisitely sensitive to the presence of acid in the bathing fluid. It was found that if the $p \mathrm{H}$ was allowed to drop by any appreciable degree the subsequent secretion of acid by that preparation was inhibited. It could be shown that standard inhibitors which acted by uncoupling oxidative phosphorylation could reduce acid secretion by a significant degree but to date it has not been possible to show stimulation of secretion by conventional stimulants such as histamine or pentagastrin. Part of the difficulty in using these stimulants is to prepare them in a buffered solution at an appropriate $p \mathrm{H}$.

It has been demonstrated that the everted rat stomach will continue to secrete acid for about four hours if the medium is unbuffered and will continue to secrete for at least seven hours at a much higher level if a buffered almost neutral solution is used.

It is suggested that the everted rat stomach is a simple preparation which may be of limited use in certain gastric secretory work, and attention is drawn to the sensitivity of the preparation to small amounts of acid in the bathing medium, an effect which cannot be mediated via the gastrin mechanism and which is hard to explain on present physiological knowledge.

RELATIONSHIP BETWEEN PH AND ABSORPTION OF ACETYLSALICYLIC ACID FROM THE STOMACH

A. R. COOKE AND J. N. HUNT Studies were made of the absorption of acetylsalicyclic acid from the stomachs of nine volunteer subjects using test meals buffered to $p H$ values of $2 \cdot 2,3 \cdot 5,7 \cdot 0$, and 9 . Quantitative studies of intragastric bleeding were made at the same time. A significant linear correlation $(P<0.001)$ was found between the amount of acetylsalicylic acid absorbed from the stomach and the $p \mathrm{H}$ of the vehicle in which the drug was dissolved, so that least absorption occurred at an alkaline or neutral $p \mathrm{H}$. Statistically significant intra- 
gastric bleeding was found only in association with absorption of acetylsalicylic acid by the stomach. The studies suggest that acetylsalicylic acid caused intragastric bleeding only if it was in a unionized state.

GASTRIC JUICE LACTIC ACIDOSIS IN GASTRIC CARCINOMA

D. W. PIPER, BARBARA H. FENTON, MERYL CROYDON, AND MARGARET KEMP A high concentration of lactic acid in the gastric juice is one of the secretory abnormalities demonstrated in the presence of gastric carcinoma. In this study the incidence of gastric juice lactic acidosis is compared in 27 cases of gastric carcinoma, 87 cases of chronic gastric ulceration, and 43 control cases. A lactic acid concentration of greater than $100 \mu \mathrm{g} / \mathrm{ml}$ was present in none of the control patients, $2.3 \%$ of gastric ulcer patients, and $60 \%$ of gastric carcinoma patients.

The effect of a lactic acid load, imposed by the intravenous administration of $100 \mathrm{~g}$ glucose, was studied as regards its effect on the lactic acid concentration of gastric juice. The imposition of a glucose load tends to accentuate the difference between the malignant and non-malignant group.

The biochemical and physiological basis of the lactic acidosis associated with gastric carcinoma was discussed.

IMMUNOGLOBULIN-SECRETING CELLS IN THE ALIMENTARY TRACT

R. S. Odgers AND A. G. WANGEL This study concerns immunoglobulin-secreting cells in the gastric mucosa of patients with pernicious anaemia or with a deficiency of iron or folate. Frozen sections prepared from biopsy specimens were fixed in acetone and stained with monovalent fluorescein-conjugated antisera against immunoglobulins A, G, and M (IgA, JgG, IgM).

In the gastric mucosa of normal subjects, patients with duodenal ulceration, and patients with folate deficiency, the majority of inflammatory cells produce $\operatorname{IgA}$ and relatively few cells produce IgG or IgM. By contrast, IgG-producing cells predominate in the gastric lesion of pernicious anaemia.

In the gastritis associated with iron deficiency, the relative proportions of immunoglobulin-producing cells appear to be related to the severity of the inflammatory process. Cells producing IgA predominate in the gastric mucosa in patients with mild histological changes. However, when the gastritis is severe, with marked cellular infiltration and loss of parietal cells, the majority of inflammatory cells produce IgG.

A comparison of biopsy specimens of rectal mucosa shows that in both normal subjects and in patients with pernicious anaemia IgA-producing cells are the most common of the cells secreting immunoglobulins. However, the normal dominance of the IgA-producing cells is greatly reduced in patients with pernicious anaemia.

GASTRIC IRON-BINDING IN HAEMOCHROMATOSIS, CIRRHOSIS, DIABETES, AND SOME DISORDERS OF IRON METABOLISM

C. G. LUKe, P. S. DAVIS, AND D. J. DELleR Previous studies have shown that gastric juice has the ability to bind iron even under alkaline conditions and that this property resides in a very high molecular weight fraction. However, gastric juice in patients with idiopathic haemochromatosis does not bind iron either at all or only to a negligible degree. Laboratory evidence has shown that this is not due to saturation of iron-binding sites by the excess iron present in this condition. Clinically, also, normal to high iron chelating ability has been demonstrated in cases of iron overload due to transfusion and chronic haemolytic anaemia.

Two of the major features of idiopathic haemochromatosis are cirrhosis and diabetes. Normal iron-binding ability has been demonstrated in both of these conditions, suggesting that the absence of gastroferrin in the idiopathic disease is not a result of either one of these features.

Evidence is presented that the gastric iron-binding substance, gastroferrin, may play a role in the luminal control of iron absorption by holding unwanted iron within the lumen of the gut. We have found that in iron deficiency less gastroferrin is produced and more iron is available for absorption. A congenital absence of gastroferrin could be a factor in the aetiology of idiopathic haemochromatosis.

\section{LACTOSE INTOLERANCE IN ASIANS}

A. E. DAVIS AND T. D. BOLIN This investigation was prompted by the clinical observation of lactose intolerance in five Asian students who were attending a gastroenterological outpatient department for vague abdominal pain associated with intermittent diarrhoea.

Lactose tolerance tests and glucose-galactose tolerance tests were performed on control subjects, Chinese students, Indian students, and a group of New Guinea natives. An extremely high incidence of lactose intolerance was found in the apparently asymptomatic Asian groups. In these groups the ingestion of lactose was associated with a flat blood sugar curve and caused abdominal pain and diarrhoea in most subjects.

There would appear to be two main aetiological factors involved. Either the defect in handling lactose is acquired due to a previously low substrate challenge or, less likely, the defect is genetically determined.

This work has two main implications. First lactose intolerance should always be considered in Asian patients complaining of abdominal pain and diarrhoea. Secondly, it has a much wider socio-economic application when it is considered the part played by milk and milk products in food aid programmes to underdeveloped Asian countries.

THE ROLE OF RADIOLOGY IN THE DIAGNOSIS OF LACTOSE INTOLERANCE IN CHILDHOOD

J. A. WALKER-SMITH AND J. D. BOWDLER Lactose intolerance is now frequently recognized in childhood. Its diagnosis is often simple in infants by stool analysis, but in older children may be more difficult. Adapting the radiological method of Laws and Neale for children, 23 barium lactose meals have been performed at the 
Royal Alexandra Hospital for Children in 23 patients, who also had lactose tolerance tests and clinical evaluation for lactose intolerance.

Three radiological appearances were seen: group I, abnormal barium lactose meal with dilution of barium, dilatation of bowel and rapid transit; group II, normal barium lactose meal; and group III, borderline abnormality, some dilution of barium, but no dilatation of bowel and normal transit. Four patients were placed in group I. All have responded to a lactose-free diet. Fourteen children fell into group II and five in group III. In these five, control barium meals without lactose were performed. In two coeliacs there was no difference from controls. In three others there was a difference.

This study suggests that barium lactose meals are a reliable screening test for lactose intolerance in childhood. If the barium lactose meal is normal no further investigation is necessary. If the barium lactose meal falls into group III, further studies-control barium meal without lactose, jejunal biopsy with enzyme assay, etc.are indicated. Such studies may also be performed to confirm the diagnosis in group I patients. However, it is considered that rapid transit, dilatation of bowel, and dilution of barium after a barium lactose meal is diagnostic of clinically significant lactose intolerance.

\section{A RAPID ORAL ISOTOPIC TEST OF CALCIUM ABSORPTION}

J. HANSKY Past methods of measuring calcium absorption have involved balance studies on a metabolic ward which are both time consuming and tedious. Recently a test utilizing $\mathrm{Ca}^{47}$ has been described by Avioli which overcomes the above objections.

Following an oral dose of 5 to $10 \mu \mathrm{c} \mathrm{Ca}{ }^{47}$ given with inert $\mathrm{CaCl}_{2}$ as carrier, plasma radioactivity was measured at 15-minute intervals for one hour and at hourly intervals for a further three hours. Urine radioactivity was concurrently determined in a four-hour sample.

In 20 normal subjects, peak plasma levels occurred between one and two hours following the dose and the levels of radioactivity attained at each period were as follows (mean $\pm \mathrm{SD}$ ): $0.76 \pm \mathbf{0 . 7 7 \%}$ dose per litre plasma at 15 minutes, $2 \cdot 20 \pm 1 \cdot 12$ at 30 minutes, $3.01 \pm$ 1.28 at 45 minutes, $3.60 \pm 1.38$ at one hour, $3.58 \pm 1.08$ at two hours, $3.10 \pm 0.78$ at three hours, and $2.54 \pm 0.58$ at four hours. Average urine radioactivity over four hours was $0.55 \pm 0.33 \%$ of administered dose. Least variation occurred in the two, three, and four-hour plasma samples in the normal population and the twohour level has been taken as the index of normal $\mathbf{C a}^{47}$ absorption. Patients with malabsorption syndrome, renal disease, and hypoadrenalism were found to have reduced calcium absorption.

These results suggest that measurement of plasma activity following oral $\mathrm{Ca}^{47}$ achieves good separation of normals from patients with abnormal calcium absorption.

THE DEMONSTRATION OF COPPER-COMPLEX FORMATION BY ENDOGENOUS ALIMENTARY CHELATES

J. L. GOllan, D. J. DEller, AND P. S. DAVIS Copper is an essential trace nutrient for man, but the mechanism controlling its absorption and excretion in health and disease is unknown.

The present study concerns the gastrointestinal absorption of copper with special reference to the interaction of this metal with alimentary secretions. Human saliva, gastric juice, pancreatic juice, and bile have been studied. The radioactive isotope $\mathrm{Cu}^{74}$ has been used in spite of its short half-life of $12 \cdot 8$ hours.

Substances were found to be present in all the abovementioned secretions which have the ability to bind copper, forming soluble complexes even at an alkaline $p \mathrm{H}$. The copper-binding substance in saliva, gastric and pancreatic juices resides in a low molecular weight fraction, as demonstrated on Sephadex G-25. Bile, in addition to a low molecular weight fraction, also binds to a high molecular weight fraction. The patterns of copper binding in saliva and gastric juice obtained by intragastric neutralization are unaltered by acid-peptic digestion.

These results suggest that in normal subjects, dietary copper is converted into low molecular weight soluble complexes before intestinal absorption. Furthermore, the pattern of copper binding in these biological fluids is quite dissimilar to that pattern of iron binding, and may well indicate differences in the mechanisms of absorption.

THE EFFECT OF VARIOUS GASTRODUODENAL HORMONES ON THE OUTPUT OF HEPATIC BILE IN THE ISOLATED PERFUSED PORCINE LIVER

M. C. DOUglas, M. Mck. WATtS, P. JABlonSKi, AND J. A. OWEN The purpose of this study is to investigate the various factors responsible for the choleresis which occurs after feeding in the intact animal.

Freshly excised porcine livers weighing between 600 and $800 \mathrm{~g}$ were perfused in a pump oxygenator circuit primed with heparinized homologous blood compatible with the blood from the hepatectomized animal. Perfusion was carried out through both hepatic artery and portal vein, the total flow rate being approximately $1 \mathrm{ml} / \mathrm{g}$ weight of liver per minute. A constant infusion of sodium taurocholate maintained the total bile acid level in the perfusate at the level found in the portal venous blood of the fasting pig. The cystic duct was ligated and hepatic bile, collected from a cannula tied into the common bile duct, was collected at regular intervals. Experiments were conducted when hepatic bile production was steady by the introduction of the test substances into the perfusate.

Neither gastrin, gastrin pentapeptide, nor cholecystokinin/pancreozymin influenced hepatic bile production in this preparation. Secretin, even in doses of less than 0.1 unit, was found to be a potent hydrocholeretic. The smallest concentration of secretin in the portal vein to produce an increase in bile flow was approximately one half the concentration necessary in the hepatic artery.

THE UPTAKE AND EXCRETION OF BROMSULPHTHALEIN (BSP) BY THE ISOLATED PIG LIVER

P. JABLONSKI, J. A. OWEN, M. C. DOUGLAS, AND J. MCK. 
WATTS The clearance of BSP by the liver has been used as a clinical test of liver function for many years. Initially, this test was applied to assess the function of isolated pig livers, perfused with either human or pig blood. It was found that clearance of the dye was rapid, but in some experiments the plasma concentration of the dye began to rise approximately $\mathbf{3 0}$ minutes after administration.

A method was developed for the estimation of conjugated and free BSP, since the liver conjugates the dye with glutathione. This estimation indicated that the reappearance of the dye could be attributed entirely to conjugated dye from the liver. When conjugated BSP was present in plasma, biliary excretion of the dye was delayed and maximum biliary concentration diminished. This phenomenon may be the result of hepatic obstruction.

In a series of experiments BSP was infused at a constant rate. Impairment of excretion and, in some cases, uptake was shown by the perfused liver. Impairment of excretion apparently precedes that of uptake from the plasma in this preparation.

The excretion of free BSP in the bile was dependent on the concentration of the free dye in the plasma: excretion of free BSP was negligible when plasma levels were very low. The percentage of biliary BSP that was in the conjugated form was apparently unaffected by the functional state of the liver, although total excretion of dye was diminished.

The effect of secretin on the excretion of BSP has been investigated. Secretin increased bile flow but biliary BSP concentrations fell. Preliminary results suggest that secretin has no effect on the uptake or excretion of BSP.

THE NATURE AND MECHANISM OF HAEMOLYSIS IN EXPERIMENTAL LIVER DISEASE

L. W. POWELL The haemolytic disorder accompanying experimental cholestatic and hepatocellular jaundice was investigated. The purpose of the study was to analyse such factors as hyperbilirubinaemia, hypersplenism and changes in osmotic fragility of red cells, which are associated with hepatobiliary disease and which might be responsible for accelerated red cell destruction.

Red cell survival was estimated following bile duct ligation in Sprague Dawley rats and Gunn rats (a Wistar strain with a congenital absence of the enzyme glucuronyl transferase) using ${ }^{51}$ chromium and $\mathrm{DF}^{32} \mathrm{P}$. A significant difference in the values for red cell survival was found between bile duct-ligated Sprague Dawley rats and their sham-operated controls, but no significant difference was observed between bile duct-ligated homozygous Gunn rats and their sham-operated controls. Heterozygous Gunn rats behaved similarly to Sprague Dawley rats with respect to haemolysis after bile duct ligation. These findings, together with an in vitro test of haemolysis, suggested that the haemolytic process accompanying cholestatic jaundice may be related to increased levels of conjugated bilirubin in the plasma, but not to the accumulation of unconjugated bilirubin, conjugated bile acids, phospholipids and cholesterol. Hypersplenism and changes in osmotic fragility of red cells did not appear to be important causative factors, but the former may have been masked by Haemobartonella muris infestation.

Results of red cell survival studies in Sprague Dawley rats treated with carbon tetrachloride were similar to those produced by bile duct ligation.

\section{CLINICO-PATHOLOGICAL CORRELATIONS IN ALCOHOLIC LIVER DISEASE}

P. M. LAST, L. J. MYKYTA, T. A. WHITE, AND M. BURR Over a period of three years we have studied 87 acknowledged heavy drinkers (mostly males) in relation to modes of presentation, socio-economic factors, clinical stigmata of cirrhosis, biochemical findings, and liver biopsy material. Histological criteria based upon those of Popper et al (Gastroenterology, 30, 357-370, 1956) were applied to form a scale comprising five grades of increasing severity of liver damage.

There were only 21 patients admitted specifically for the treatment of various manifestations of alcoholism, and there were 43 in whom a drinking problem was apparently not suspected by the referring practitioner.

Sixty of the 87 patients had hepatomegaly, and in 31 of these there was no other abnormality. Twenty-seven patients had no significant physical findings. Physical stigmata of chronic liver disease were uncommon.

Conventional laboratory studies were generally unhelpful, with the exception of the electrophoretic pattern, but even with this test the correlation between biochemical and histological degrees of severity was poor. The best reflections of significant liver damage were the serum albumin level, which was lowest in the most advanced cases, and the ' $\beta-\alpha$ veil', which was found to be present only with severe degrees of hepatic damage.

Histological grading showed a surprising total of 47 cases with advanced liver damage beyond that expected from clinical or biochemical findings.

Eleven patients had advanced liver disease shown on biopsy: none of them had any physical signs of biochemical abnormality whereby the degree of damage could have been suspected.

Heavy alcoholic consumption is common in the average Australian population. In this study we found evidence of significant hepatic damage on liver biopsy in over half of our patients. Clinical and biochemical assessment proved disappointingly unreliable, and only by means of biopsy can the severity of the hepatic damage be adequately assessed.

\section{CLINICAL MEETING}

A clinical meeting was held on 28 May. It included panel discussions on haemochromatosis and ZollingerEllison syndrome. 